\section{Federation University ResearchOnline}

\section{https://researchonline.federation.edu.au}

Copyright Notice

This paper is a postprint of a paper submitted to and accepted for publication in 2014 IEEE $16^{\text {th }}$ International Workshop on Multimedia Signal Processing. The copy of record is available at the IET Digital Library.

Chakraborty, Paul, M., Murshed, M., \& Ali, M. (2014). A novel video coding scheme using a scene adaptive non-parametric background model. 2014 IEEE 16th International Workshop on Multimedia Signal Processing (MMSP), 1-6.

Available online: https://doi.org/10.1109/MMSP.2014.6958823

Copyright @ 2014 IEEE

See this record in Federation ResearchOnline at: http://researchonline.federation.edu.au/vital/access/HandleResolver/1959.17/161507 


\title{
A Novel Video Coding Scheme using a Scene Adaptive Non-Parametric Background Model
}

\author{
Subrata Chakraborty ${ }^{* 1}$, Manoranjan Paul ${ }^{* 2}$, Manzur Murshed ${ }^{\# 3}$, Mortuza Ali ${ }^{\# 4}$ \\ *School of Computing \& Mathematics, Charles Sturt University, NSW 2795, Australia \\ schakrabortylesu.edu.au, \\ 2 mpaulecsu.edu. au \\ \# School of Information Technology, Federation University, Gippsland, VIC 3842, Australia \\ ${ }^{3}$ Manzur.Murshedefedaration.edu.au, \\ ${ }^{4}$ Mortuza.Ali@fedaration.edu.au
}

\begin{abstract}
Video coding techniques utilising background frames, provide better rate distortion performance by exploiting coding efficiency in uncovered background areas compared to the latest video coding standard. Parametric approaches such as the mixture of Gaussian (MoG) based background modeling has been widely used however they require prior knowledge about the test videos for parameter estimation. Recently introduced non-parametric (NP) based background modeling techniques successfully improved video coding performance through a HEVC integrated coding scheme. The inherent nature of the NP technique naturally exhibits superior performance in dynamic background scenarios compared to the MoG based technique without a priori knowledge of video data distribution. Although NP based coding schemes showed promising coding performances, they suffer from a number of key challenges - (a) determination of the optimal subset of training frames for generating a suitable background that can be used as a reference frame during coding, (b) incorporating dynamic changes in the background effectively after the initial background frame is generated, (c) managing frequent scene change leading to performance degradation, and (d) optimizing coding quality ratio between an $I$-frame and other frames under bit rate constraints. In this study we develop a new scene adaptive coding scheme using the NP based technique, capable of solving the current challenges by incorporating a new continuously updating background generation process. Extensive experimental results are also provided to validate the effectiveness of the new scheme.
\end{abstract}

\section{INTRODUCTION}

In video coding domain providing better quality video at a lower bit rate is still an open issue. Finding the right balance is the key to provide the best possible quality for the available bit rate. With the advancement of technology and the availability of high speed networks, larger bandwidths currently exist. However, sophisticated video capturing, display technologies, and incremental users' demand on higher resolution and quality also produce large sized video data. Hence the challenge to find the appropriate balance between quality and bit rate still remains.

The latest HEVC video coding standard [1] improved the coding performance by applying a number of innovative tools compared to its predecessor H.264/AVC [2] including a wider range of variable block size motion estimation (ME), motion compensation (MC), prediction, and transformation units. The use of multiple reference frames (MRFs) using variable block sizes tools typically provides better coding performance than single reference frame approach [1][3]. However, MRFs schemes require index codes to identify a particular reference frame and the computational time increases almost linearly with the each additional reference frames due to ME \& MC. The decision on appropriate number of reference frames is dependent on the video content and the computational time constraint may not always allow large number of reference frames [4][5]. Dual reference frame based schemes [6][7] solves the challenges in MRFs by using the previous frame as the short term reference (STR) for ME \& MC and a stable frame (such as background) as long term reference (LTR)

Various fast coding approaches [8][11] achieved significant time saving compared to $\mathrm{H} 264$ but failed to outperform it in rate-distortion (RD) performance for video sequences with repetitive motion, newly uncovered background, non-integer pixel displacement, lighting change, etc. [4]. Video segmentation oriented approaches investigated into exploiting the comparatively stable parts in a frame by treating them as backgrounds [12][14]; however they require high computational time. Sprite coding schemes based on object segmentation [15][16] were also introduced but they suffer from high computational burden with their performance degrading for high bit rate applications [17].

McFIS (most common frame in scene) based coding schemes [4][5][18][19] were introduced to utilize the highly accepted Mixture of Gaussian (MoG)-based dynamic background modeling (DBM) techniques [20][21]. The coding schemes using the McFIS further highlighted the fact that using a good quality background frame as a reference frame improves coding performance and computational efficiency for mostly static and uncovered background areas compared to the coding scheme with MRFs using a number of previously decoded frames. The need for using a good DBM technique for practical usage is also highlighted. Studies have shown the improved performance and computational efficiency of the McFIS-based video coding schemes [4][5][18][19][22]. DBM is also applied in recent studies in the area of transcoding technique for video surveillance [23].

The MoG based DBM works at pixel level with each pixel of a scene is modeled independently using a mixture of $\Psi$ (generally 3 to 5) Gaussian distributions [20][22][24][25]. 
Despite the MoG based DBM techniques have been proved successful and widely used by researchers and practitioners, they require the user to assume the data distribution in advance and relevant parameters must be set based on this underlying assumption. It is also found to be performing poorly for fast changing background environments [24][26]. A relatively new non-parametric (NP) techniques [24][26][27] has gained the attention of many researchers due to its ability to perform well in highly dynamic scenarios which requires no initial assumption about the underlying data distribution [24][26][28]. The NP technique uses historical pixel values for a given pixel to perform probability density estimation using kernel estimator $K$ [24][26]. The background model for a frame is developed by comparing the probability of the current intensity value of each pixel against a threshold (to decide whether background or foreground) [24][26]. Although the high sensitivity to dynamic background environment makes the NP technique very attractive for applications such as object detection and tracking, this poses a serious challenge for video coding. If the background is updated more frequently then the coding of more background frames would be necessary. This may lead to a higher bit rate requirement. Moreover, in the existing NP technique [24][26], the background is generated using the pixel intensity values of the last frame only and the historical pixel intensity values are only used for probability estimation purpose. Hence, the background is heavily biased to the last frame and loses the historical trend value. This might provide a good background frame for detecting an object for the current frame for object detection applications; however, as an extra reference frame, it does not contribute significantly in video compression of the current frame as the last frame has normally been used for $\mathrm{ME}$ and MC of the current frame in HEVC or H.264. To resolve these issues recently a weighted non-parametric (WNP) technique was introduced [29] where a comparatively stable background was generated using historical pixel values and the latest pixel value of the latest frame. The WNP technique uses the weighted average of (i) a probabilistic pixel intensity value (calculated based on median of historical values and randomly scaled standard deviation) and (ii) the latest pixel intensity value. This process scales the actual pixel intensity value by maintaining the historical trend of the intensities of the pixel position in the generated background. The background remains more stable, thus reducing the frequent background updating issue with NP.

Although the WNP technique showed impressive RD coding performances against HEVC, MoG and traditional NP based coding schemes [29], there are several practical challenges still exists due to its current coding scheme. In the current coding scheme using WNP a background is generated using a set of training frames which is then coded as an Iframe to be used as a reference frame while coding future frames. The $\boldsymbol{1}^{\text {st }}$ challenge is to determine the size of the training frame set. A user must make sure the size is large enough to produce a good quality background. If the size is too large it will affect the coding performance as HEVC is used as the default coder during the background generation process. On the other hand if the size is too small a useful background may not be produced leading to performance degradation. The required training set size is also largely dependent on the video contents. A video with highly dynamic background will require a larger size whereas a video with static background and low object movement will need smaller size of training set to capture the background. In the current WNP based scheme once the background is generated it is then used as a reference to code the following stream of frames. The background reference frame is only reproduced when a scene change is detected. This process highlights the $2^{\text {nd }}$ challenge that if there are subtle changes such as newly exposed previously occluded areas, the current scheme is not capable of capturing that change. The $3^{\text {rd }}$ challenge of the existing WNP based scheme relates to the scene change handling. If there are frequent scene changes, in the current scheme a new background frame needs to be generated for each change and then has to be coded as an I-frame which generally requires more bits leading to performance degradation. The $4^{\text {th }}$ Challenge of the current WNP based scheme [29] is deciding the quantization ratio between an $I$ frame and a normal frame. If there are a large numbers of scene changes in a video, setting a lower quantization value for the $I$-frames will lead to higher bit rate requirements leading to $\mathrm{RD}$ performance degradation. On the contrary higher quantization will decrease PSNR leading to RD performance degradation. Finding the right quantization ratio in the current scheme is thus a persistent challenge as it depends on the video content.

In this study we propose a novel Scene Adaptive NonParametric (SANP) coding scheme which adopts the WNP [29] for background modelling. In this scheme we use the code frames instead of original input frames. The background reference frame is iteratively updated with each new coded frame thus resolving the challenges 1 and 2 . The proposed SANP scheme eliminates the need for $I$-frame coding by its inherent architecture thus solves the challenges 3 and 4.

In section II we present the proposed SANP scheme followed by detailed experimental results and comparative discussions in section IV.

\section{Proposed Scene AdAPtive Coding Scheme}

The SANP technique uses dual reference [6][7] frames technique where the LTR is a high quality background frame (generated by the proposed SANP background modeling) and the STR is the immediate previous frame of the current frame. The background frame is modeled with a small set of coded frames of a scene and used as a LTR frame. The LTR frame is updated each time a newly coded frame is available. All frames in the scene are encoded using dual reference frames. The Lagrangian RD cost function of the HEVC is used to finally select the reference frame for a block or sub-block.

Fig. 1. shows the SANP coding scheme. In this scheme the initial set of fames are coded using the standard HEVC coder. The coded frames are then used by the SANP background generator to generate a background frame to be used as a reference frame by the McFIS based coder for coding the next 
new frame. Each coded frame is then used by the SANP background generator to develop a new background frame to be used as reference for coding the next frame. In SANP the background frame gets updated with each new coded frame.

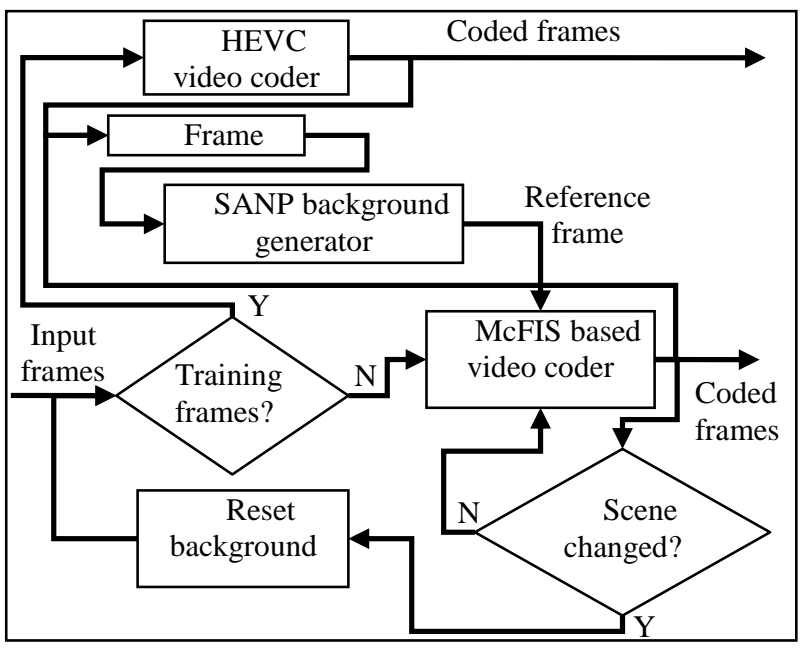

Fig. 1. SANP coding scheme

A scene change detection strategy similar to [5] is also adopted in the proposed SANP scheme. When a scene change occurs we reset the background modeling and generate a new background frame for the new scene. The SANP technique is primarily based on the WNP [29] and utilizes the traditional NP Technique [24][26][27] for background detection. The SANP generates a stable background frame from a set of coded frames integrating both the historical pixel intensity value and the recent pixel intensity value from the last training frame for each pixel. Considering a combination of the pixel intensity of the latest frame and the historical pixel intensity provides better RD performance in video coding applications [29]. Background frame comprising historical pixel intensities ensures overall minimization of residual error of all frames, resulting in better overall compression.

The SANP background modeling and background generation technique is described in the following 2 stages which mimics WNP [29]:

Stage 1: Background probability estimation technique ([24][26][27])

Assume $x_{1}, x_{2}, \ldots, x_{N}$ is a set of recent intensity values for a pixel. The probability density function with pixel intensity $x_{t}$ at time $t$ by using the kernel estimator $K($.) as

$$
p_{r}\left(x_{t}\right)=\frac{1}{N} \sum_{i=1}^{N} K\left(x_{t}-x_{i}\right)
$$

where $K($.$) is a kernel estimation function. If we consider that$ the kernel estimator function $K($.) to be a Normal function $N(0, \Sigma)$ where $\sum$ is the kernel function bandwidth. With the assumption of independence between the different colour channels $d$ and with different kernel bandwidths $\sigma_{j}^{2}$ for the $j$ th colour channel, the density estimation equation can be

$$
p_{r}\left(x_{t}\right)=\frac{1}{N} \sum_{i=1}^{N} \prod_{j=1}^{d} \frac{1}{\sqrt{2 \pi \sigma_{j}^{2}}} e^{-\frac{1}{2} \frac{\left(x_{t j}-x_{i_{j}}\right)^{2}}{\sigma_{j}^{2}}}
$$

In order to estimate the kernel bandwidth $\sigma_{j}^{2}$ for $j$ th colour channel for any given pixel the median absolute deviation $m$ over the sample for consecutive intensity values of the pixel calculated as $m=$ median $\left(\left|x_{i}-x_{i+1}\right|\right)$ where $i=1,2, \ldots, N-1$. We can consider that the intensity pair being consecutive comes from local-in-time distribution. Assuming that the local-in-time distribution is Normal $N\left(\mu, \sigma^{2}\right)$, then the deviation $\left(x_{i}-x_{i+1}\right)$ is also Normal. The standard deviation of the first distribution can be computed as $\sigma=m /(0.68 \sqrt{2})$.

Stage 2: Background generation

In the proposed SANP we mimic the generic background generation process of the WNP technique [29]. The background is determined using a $20 \%$ variation threshold recommended in [26]. If the current pixel value $x_{t}$ is detected as background, the value is combined with the synthesized historical pixel intensity of the given sample for that particular pixel. The synthesized historical pixel value $M$ for the sample pixel values can be calculated as

$$
M=\text { median }\left(x_{1}, x_{2}, \ldots, x_{N}\right)+\sigma^{*} Y
$$

The synthesized value $M$ is a mimic of the actual pixel intensity generated using the standard deviation and a very small normally distributed random multiplier $Y$ which can generated using the randn(.) function in Matlab which generates a normally distributed random value. This is then used to generate the synthesised pixel value considering the calculated median as mean and using the standard deviation $\sigma$.

The value $X$ of the background pixel is calculated as a weighted average of actual value $x_{t}$ and synthesized value $M$ :

$$
X=x_{t} * \alpha+M *(1-\alpha)
$$

The SANP background generation process defines a size for a frame set required for generating the background using (3) and (4). This set is iteratively updated with each coded frame as shown in Fig. 1.. Let us assume that the set size is determined to be 25 frames. At the beginning HEVC coder is used to code the first 25 frames which are then sent to the frame buffer (size 25). When the buffer is full, the WNP based background generator is used to generate the background frame which is then used by the McFIS coder to code frame no 26. The coded $26^{\text {th }}$ frame is then sent to the frame buffer and the $1^{\text {st }}$ frame is discarded from the buffer. A new background is then generated from the frames in the buffer. The new background reference frame is generated by taking the average of the current reference frame and the newly created background frame. This process is repeated for each new coded frame where the oldest frame in the buffer is replaced with the new frame in a first-in-first-out sequence.

Let us consider that the background frame generated by the SANP background generator is denoted as $G$ with width $y$ and 
height $z$. Each pixel $X$ in frame $G$ can be calculated applying (4). Each pixel in the background frame can be referred as $X_{G, y, z}$. Initially this background frame $G$ is the reference frame $R$. Each pixel of the reference frame can be obtained as:

$$
X_{R, y, z}=X_{G, y, z}
$$

With each newly coded frame a new background frame $G$ is generated by SANP background generator. The reference frame $R$ is updated with each new coded frame by updating the pixels of the reference frame as:

$$
X_{R, y, z}=\left(X_{R-1, y, z}+X_{G, y, z}\right) / 2
$$

The SANP scheme maintains the stability of the background reference frame by incorporating the pixel values of all the past background frames in the new reference frame. At each iteration the impact of past frames is reduced by a rate of $e^{-2}$, thus providing more importance to recent reference frames to achieve adaptation to background changes.

When a scene change is detected the background reference frame is reset to initial state. HEVC coder is used until another background reference frame is generated. The SANP scheme eliminates the need for $I$-frame coding as the scheme can be used simultaneously at both the coding and decoding end of the coder. The continuous updating of the background reference frame makes it adaptive to changes in the input frame thus improving the coding performance.

Selection process of $\alpha$ : The value of $\alpha$ in (4) is adaptively adjusted to provide more importance to either the recent value or the historical value trend for a given pixel. An adaptive procedure described in 6 steps [29]:

\section{1) Decide the potential $\alpha$ values}

At this stage we decide a set of potential $\alpha_{p}$ where $p=1 . . P$ is the number of $\alpha$ values we are interested in for a given video dataset. The $\alpha$ values are between 0 and 1 and may be selected at regular intervals.

2) Generate background for each $\alpha$

A background frame is generated for each $\alpha$ by applying the background generation method described earlier. We can denote the backgrounds as $B_{p}$ where $p=1 \ldots P$.

\section{3) Calculate pixel intensity variation}

With the assumption of $F_{q}$ where $q=1 \ldots Q$ training frames with width $W$ and height $H$, we find the intensity difference $I$ for each pixel of each frame with the corresponding pixel for each background can be calculated as:

$$
I_{p q}(w, h)=\left|B_{p}(w, h)-F_{q}(w, h)\right|
$$

where $p=1 . . P ; q=1 . . Q ; w=1 . . W ; h=1 . . H$

\section{4) Count background pixel detection}

The background detection percentage represents the area of a frame detected as background by the SANP method using a particular $\alpha$ value. The total number of pixels in a frame is $W^{*} H$. The number of pixels detected as background in a frame using a particular $\alpha$ value can be calculated by (7) as

$$
C_{p q}=\operatorname{Count}\left(I_{p q}(w, h)<=\delta\right)
$$

The small value $\delta$ is the threshold for a pixel to be considered as background. Ideally, the value of $\delta$ should be " 0 ". However the SANP develops a synthesized background using historical and actual pixel values which leads to deviation from the actual pixel value and thus a nonzero $\delta$ value for some cases. With a significant amount of experimental studies using test videos is was identified that generally a large change in the pixel value classifies it as foreground pixel, while only a small change classifies it as background pixel. Experimental results indicate that up to $2 \%$ deviation from the highest possible pixel value (i.e. 255) may be used as the $\delta$ value [29].

\section{5) Calculate background detection percentage}

By applying (8) calculate the percentage of total pixels detected as background for a particular frame by:

$$
U_{p q}=\left(C_{p q} * 100\right) /(W * H)
$$

The overall background detection percentage for a test video is calculated based on the number of training frames $F_{q}$. The overall background detection $\alpha_{p}$ can be calculated as:

$$
U_{p}=\left(\sum_{q=1}^{Q} U_{p q}\right) / Q
$$

\section{6) Select $\alpha$ for a video}

The $\alpha$ value for a video is $\alpha_{p}$ for the maximum $U_{p}$.

$$
\alpha=\alpha_{p} \text { for } \max \left(U_{p}\right) \text { where } p=1,2, . . P
$$

In the following section we present experimental results and analyses to prove the significance of the SANP scheme.

\section{EXPERIMENTAL RESULTS AND ANALYSES}

\section{(a) Experiment setup}

The experiments were conducted on a dedicated desktop machine (with Intel core i7 $3770 \mathrm{CPU} @ 3.4 \mathrm{GHz}, 16 \mathrm{~GB}$ RAM and 1TB HDD) running 64 bit Windows operating system. The program codes for the NP and WNP techniques were available from the authors. We have compared WNP and SANP over several test videos. For further comparison we used the traditional NP, MoG with an adaptive scheme similar to the SANP scheme for coding-decoding and compared with performances of the standard HEVC and SANP scheme. For HEVC we used GOP of size 30. For Inter coding we used 2 reference frames. Motion search was performed within a window of \pm 31 . For NP, MoG and WNP, the generated background frame by each technique was used as a reference frame along with the immediate previous frame for codingdecoding purpose. The test video sequences Sales, News, Grandma and Table Tennis are of resolution of $176 \times 144$, while the Silent, Paris and Container test video sequences are of resolution of $352 \times 288$. First 25 frames were used as training frames of a scene to generate the background frame for the MoG, NP and WNP techniques.

For the original input frame based WNP scheme the initial total training frames were chosen to be 25 because provides a buffering time of $<1$ second at standard 30fps. This is acceptable for most of the applications. A set of 25 frames 
was also large enough size to provide a good quality background for all our test video sequences. The background frame was then coded as $I$-frame. For the WNP technique we have applied the $\alpha$ selection procedure described earlier to select appropriate $\alpha$ for each video sequence.

For the SANP scheme, we have coded first 25 frames using HEVC. These coded frames were used to generate the reference background frame. For NP and MoG similar coding scheme was applied where the background was generated and continuously updated using the coded frames.

We have used 5 different quantization values $(\mathrm{QP}=28,24$, 20,16 , and 12) for each sequence to obtain different bit rates and the corresponding PSNR values. Then each of the four coding techniques for each scheme was used to code-decode the video sequences.

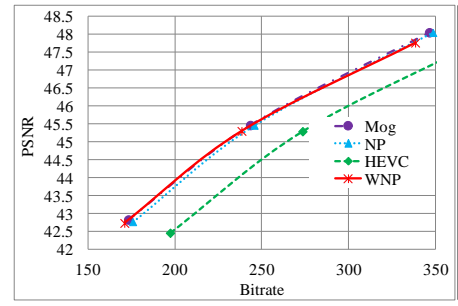

(a) WNP

Fig. 2. RD performance graphs for News video

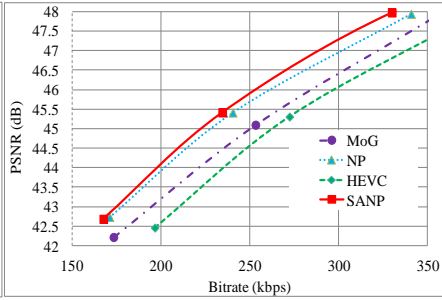

(b) SANP

\section{(b) Results and discussions}

In order to understand the efficiency let us consider the News video. The News video contains dynamic background (ballet dancing in the background) behind the objects (two news readers). This is a particularly challenging video as the background keeps changing over time and requires it to be distinguished from the objects. A performance comparison is shown in Fig. 2. If we look carefully at Fig. 2 (a), the current WNP and NP using non-adaptive scheme performs very similar to the MoG based scheme and even worse in high bit rate. The poor performance of WNP is due to its existing non adaptive scheme as the initial background reference frame is not updated with changes in the background. On the contrary both the new adaptive SANP and adaptive NP perform much better than the adaptive MoG as well as the standard HEVC. The SANP scheme well and truly exploits the dynamic background handling capabilities of the NP technique thus convincingly outperforms the MoG as shown in Fig. 2 (b).

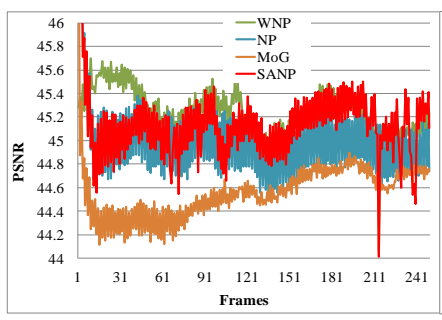

(a) Sales video

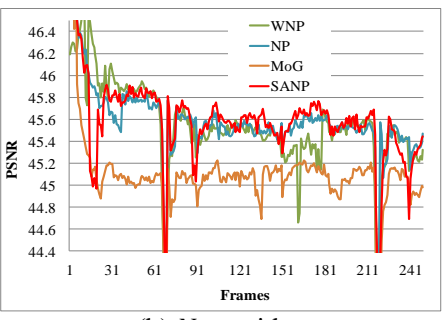

(b) News video
Fig. 3. Frame by Frame PSNR

To understand the concept further we look at frame by frame coding performances. Fig. 3 shows frame by frame PSNR at comparable bit rates for the SANP, WNP, adaptive
MoG and adaptive NP based schemes. We will look closely at the Sales video which contains highly static background and the News video as which contains highly dynamic background. For the Sales video in Fig. 3 (a) the SANP and WNP performs similarly and better than NP and MoG. We can observe that although WNP was performing better than SANP at the beginning its performance degraded for distant frames. This is due to the fact that WNP scheme does not adapt to the changes in the background. SANP on the other hand updates the background reference frame continuously resulting in better performance throughout. For the dynamic News video Fig. 3 (b) SANP shows the best performance trend over the frame set. This confirms the superior overall performance of SANP presented earlier in Fig. 2. We have also used our adaptive scheme for traditional NP technique resulting in improved performances. The existing WNP may perform well when the background generated at the beginning remains stable throughout the video or when no new background area is exposed later in the video. The SANP performs very well in most of the videos including dynamic ones and eliminates the current challenges of WNP through its inherent scheme architecture.

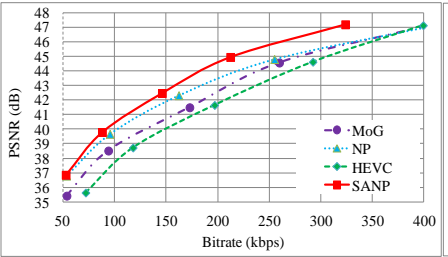

(a) Sales video

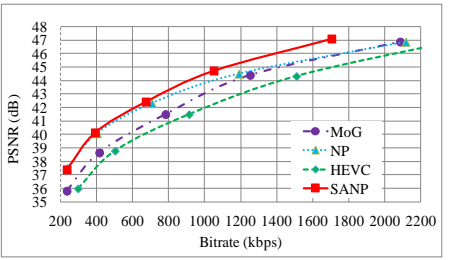

(b) Silent video
Fig. 4. RD performances for tested techniques

Performance curves for the Sales and Silent videos are presented in Fig. 4. We can observe that the proposed SANP outperforms the standard HEVC, adaptive MoG and the adaptive NP schemes. Table I shows the coding performances of the new SANP scheme along with HEVC, adaptive schemes with NP and MoG background modelling techniques at different bit rates for the set of test videos. For the Sales video on average the SANP outperforms HEVC, adaptive NP and adaptive MoG by $2.3 \mathrm{~dB}, 1 \mathrm{~dB}$ and $1.7 \mathrm{~dB}$ respectively. For the News video on average the SANP outperforms HEVC, adaptive $\mathrm{NP}$ and adaptive $\mathrm{MoG}$ by $1.5 \mathrm{~dB}, 0.2 \mathrm{~dB}$ and $0.9 \mathrm{~dB}$ respectively. For the Silent video on average the SANP outperforms HEVC, adaptive NP and adaptive MoG by $2.3 \mathrm{~dB}, 0.85 \mathrm{~dB}$ and $1.3 \mathrm{~dB}$ respectively. For the Paris video on average the SANP outperforms HEVC, adaptive NP and adaptive MoG by $0.95 \mathrm{~dB}, 0.45 \mathrm{~dB}$ and $1.2 \mathrm{~dB}$ respectively. For the Grandma video on average the SANP outperforms HEVC, adaptive NP and adaptive MoG by $1.1 \mathrm{~dB}, 0.35 \mathrm{~dB}$ and $0.85 \mathrm{~dB}$ respectively. For the Table video on average the SANP outperforms HEVC, adaptive NP and adaptive MoG by $0.55 \mathrm{~dB}, 0.8 \mathrm{~dB}$ and $0.2 \mathrm{~dB}$ respectively. For the Container video on average the SANP outperforms HEVC, adaptive NP and adaptive MoG by $0.5 \mathrm{~dB}, 0.4 \mathrm{~dB}$ and $0.15 \mathrm{~dB}$ respectively. The results confirm the efficiency of the SANP scheme. 
Table I

PSNR AT DIFFERENT BIT RATES FOR THE TESTED SCHEMES

\begin{tabular}{|c|c|r|r|r|r|}
\hline \multirow{2}{*}{ Videos } & $\begin{array}{c}\text { Bit rate } \\
(\mathrm{kbps})\end{array}$ & HEVC & NP & MoG & SANP \\
\hline \multirow{2}{*}{ Sales } & 150 & 40.00 & 41.90 & 40.70 & $\mathbf{4 2 . 7 0}$ \\
\cline { 2 - 6 } & 300 & 44.90 & 45.60 & 45.40 & $\mathbf{4 6 . 8 0}$ \\
\hline \multirow{2}{*}{ News } & 100 & 37.90 & 39.40 & 38.60 & $\mathbf{3 9 . 5 0}$ \\
\cline { 2 - 6 } & 250 & 44.50 & 45.60 & 45.00 & $\mathbf{4 5 . 9 0}$ \\
\hline \multirow{2}{*}{ Silent } & 1000 & 42.00 & 43.80 & 43.00 & $\mathbf{4 4 . 5 0}$ \\
\cline { 2 - 6 } & 1600 & 44.70 & 45.80 & 45.70 & $\mathbf{4 6 . 8 0}$ \\
\hline \multirow{2}{*}{ Paris } & 1400 & 41.20 & 41.90 & 40.90 & $\mathbf{4 2 . 4 0}$ \\
\cline { 2 - 6 } & 2400 & 44.80 & 45.10 & 44.60 & $\mathbf{4 5 . 5 0}$ \\
\hline \multirow{2}{*}{ Grandma } & 150 & 41.40 & 42.60 & 41.70 & $\mathbf{4 2 . 9 0}$ \\
\cline { 2 - 6 } & 350 & 46.10 & 46.40 & 46.30 & $\mathbf{4 6 . 8 0}$ \\
\hline \multirow{2}{*}{ Table } & 500 & 44.20 & 43.90 & 44.80 & $\mathbf{4 4 . 9 0}$ \\
\cline { 2 - 6 } & 800 & 48.00 & 47.80 & 48.10 & $\mathbf{4 8 . 4 0}$ \\
\hline \multirow{2}{*}{ Container } & 1500 & 42.8 & 42.90 & 43.20 & $\mathbf{4 3 . 3 0}$ \\
\cline { 2 - 6 } & 2500 & 45.90 & 46.00 & 46.20 & $\mathbf{4 6 . 4 0}$ \\
\hline
\end{tabular}

\section{CONCLUSION}

A novel scene adaptive coding scheme using the WNP background modelling technique has been presented in this study. The proposed SANP technique adopts the strengths of the non-parametric background modelling technique including automated parameter estimation and better dynamic background detection. The new scheme eliminates several challenges with the existing WNP technique by integrating an adaptive reference background frame generation process. The stability of the reference background frame and capacity to incorporate new changes in the background makes SANP more efficient for dynamic videos while performing very well for more static videos. The WNP may perform very well with highly static non changing backgrounds if the initial training set is large enough to produce near perfect background. The SANP performs as good as WNP for videos static background but shows much better performances for challenging dynamic background videos. We have also applied the adaptive scheme for traditional NP background modelling thus improving its performance for dynamic videos. The new scheme would provide researchers and practitioners an efficicient and dynamic option which can be applied for coding a wide range of videos.

\section{REFERENCES}

[1] G.J. Sullivan, J.-R. Ohm, W.-J. Han, and T. Wiegand, "Overview of the High Efficiency Video Coding (HEVC) Standard," IEEE Trans. Circuits Syst. Video Technol. (TCSVT), 22(12), 1649-1668, 2012.

[2] T. Wiegand, G. J. Sullivan, G. Bjøntegaard, and A. Luthra, "Overview of the H.264/AVC Video Coding Standard," IEEE TCSVT, 13(7), 560-576, 2003.

[3] J. -R. Ding and J. -F. Yang, "Adaptive group-of-pictures and scene change detection methods based on existing H.264 advanced video coding information," IET Image Process., 2(2), 85-94, 2008.

[4] M. Paul, W. Lin, C. T. Lau, and B. -S. Lee, "McFIS: better I-frame for video coding," IEEE ISCAS-10, pp. 2171-2174, 2010.

[5] M. Paul, W. Lin, C. T. Lau, and B. -S. Lee, "Explore and model better Iframes for video coding," IEEE TCSVT, 21(9), 1242-1254, 2011.

[6] D. Liu, D. Zhao, X. Ji, and W. Gao, "Dual Frame Motion Compensation With Optimal Long-Term Reference Frame Selection and Bit Allocation," IEEE TCSVT, 20(3), 325 - 339, 2010.
[7] A. Mavlankar and B. Girod, "Background extraction and long-term memory motion-compensated prediction for spatial-random-accessenable video coding," Int. Picture coding Symposium, 2009.

[8] Y. -W. Huang, B. -Y. Hsieh, S. -Y. Chien, S. -Y. Ma, et al., "Analysis and complexity reduction of multiple reference frames motion estimation in H.264/AVC," IEEE TCSVT,.16(4), 507-522, 2006.

[9] L. Shen, Z. Liu, Z. Zhang, and G. Wang, "An Adaptive and Fast Multi frame Selection Algorithm for H.264 Video Coding," IEEE Signal Process. Lett., vol. 14, No. 11, pp. 836-839, 2007.

[10]T. -Y. Kuo, H. -J. Lu, "Efficient Reference Frame Selector for H.264," IEEE TCSVT, 18(3), 400-405, 2008.

[11]K. Hachicha, D. Faura, O. Romain, and P. Garda, "Accelerating the multiple reference frames compensation in the H.264 video coder", Journal of Real-Time Image Processing, 4(1)1, 55-65, 2009.

[12]D. Hepper, "Efficiency analysis and application of uncovered background prediction in a low bit rate image coder," IEEE Trans. on Commun., vol. 38, pp. 1578-1584, 1990.

[13]S. -Y. Chien, S. -Y. Ma, and L. -G. Chen, "Efficient Moving Object Segmentation Algorithm Using Background Registration Technique," IEEE TCSVT., 12(7), 577-586, 2002.

[14]T. Totozafiny, O. Patrouix, F. Luthon, and J. -M. Coutellier, "Dynamic Background Segmentation for Remote Reference Image Updating within Motion Detection JPEG2000," IEEE Int. Symp. on Industrial Electronics, vol. 1, pp.505-510, 2006.

[15] T. Sikora, "Trends and perspectives in image and video coding," Proc. IEEE, vol. 93, no. 1, pp. 6-17, 2005.

[16]M. Kunter, P. Krey, A. Krutz, and T. Sikora, "Extending H.264/AVC with a background sprite prediction mode," IEEE Int. Conf. on Image Process. (ICIP 08), pp. 2128-2131, 2008.

[17] R. Ding, Q. Dai, W. Xu, D. Zhu, and H. Yin, "Background-frame based motion compensation for video compression," IEEE Int. Conf. on Multimedia and Expo (ICME 04), vol. 2, pp. 1487-1490, 2004.

[18] M. Paul, W. Lin, C. T. Lau, and B. -S. Lee, "McFIS in hierarchical bipredictve pictures-based video coding for referencing the stable area in a scene," IEEE ICIP 11, 3521-3524, 2011.

[19]M. Paul, W. Lin, C. T. Lau, and B. -S. Lee, "Video coding using the most common frame in scene," IEEE Int. Conf. on Acoustics, Speech, and Signal process. (ICASSP 10), pp.734-737, 2010.

[20]C. Stauffer and W. E. L. Grimson, "Adaptive background mixture models for real-time tracking," IEEE Conf. on Computer Vision and Pattern Recognition, vol. 2, pp. 246-252, 1999.

[21]D.-S. Lee, "Effective Gaussian mixture learning for video background subtraction," IEEE Trans. on Pattern Analysis and Machine Intelligence, vol. 27, no. 5, pp. 827-832, 2005.

[22] M. Paul, W. Lin, C. T. Lau, and B. -S. Lee. "Video coding with dynamic background" EURASIP Journal on Advances in Signal Processing, vol. 2013, no.1, pp. 1-17, 2013.

[23]M. Geng, X. Zhang, Y. Tian, L. Liang, and T. Huang, "A Fast and Performance-Maintained Transcoding Method Based on Background Modeling for Surveillance Video," IEEE Int. Conf. on Multimedia and Expo (ICME 12), pp.61-66, 2012.

[24]A. Elgammal, D. Harwood, and L. Davis, "Non-parametric model for background subtraction," ECCV, pp. 751-767, 2000.

[25] M Haque, M Murshed, and M Paul, "Improved Gaussian mixtures for robust object detection by adaptive multi-background generation," IEEE Int. Conf. on Pattern Recognition, pp. 1-4, 2008.

[26]A. Elgammal, R. Duraiswami, D. Harwood, and L. S. Davis, "Background and foreground modeling using nonparametric kernel density estimation for visual surveillance," Proc. IEEE, vol. 90, no. 7, pp. 1151-1163, 2002

[27]A. Elgammal, "Background Subtraction: Theory and Practice," Springer, 2013.

[28]Z. Zivkovic and F. van der Heijden, "Efficient adaptive density estimation per image pixel for the task of background subtraction", Pattern Recognition Letters, vol. 27, no. 7, pp. 773-780, 2006.

[29] S. Chakraborty, M. Paul, M. Murshed, and M. Ali, "IEEE International conference on Multimedia and Expo (IEEE ICME-14), 2014. 